\title{
THE IMAGE REGISTRATION OF FOURIER-MELLIN BASED ON THE COMBINATION OF PROJECTION AND GRADIENT PREPROCESSING
}

\author{
Gao Dongyang ${ }^{\mathrm{a}}$, Zhao Xian ${ }^{\mathrm{b}}$, Pan Xin $^{\mathrm{c}, *}$ \\ ${ }^{a}$ School of Geomatics and Urban Spatial Information, Beijing University of Civil Engineering and Architecture, 100044 Beijing, \\ China-574273129@qq.com \\ ${ }^{\mathrm{b}}$ School of Geomatics and Urban Spatial Information, Beijing University of Civil Engineering and Architecture, 100044 Beijing, \\ China \\ ${ }^{\mathrm{c}}$ School of Geomatics and Urban Spatial Information, Beijing University of Civil Engineering and Architecture, 100044 Beijing,
} China-panxin@bucea.edu.cn

\section{Commission III, WG III/6}

KEY WORDS: Non-parallel image pairs, Projection transformation, gradient preprocessing, Fourier-Mellin transformation, image registration

\begin{abstract}
:
Image registration is one of the most important applications in the field of image processing. The method of Fourier Merlin transform, which has the advantages of high precision and good robustness to change in light and shade, partial blocking, noise influence and so on, is widely used. However, not only this method can't obtain the unique mutual power pulse function for non-parallel image pairs, even part of image pairs also can't get the mutual power function pulse. In this paper, an image registration method based on Fourier-Mellin transformation in the view of projection-gradient preprocessing is proposed. According to the projection conformational equation, the method calculates the matrix of image projection transformation to correct the tilt image; then, gradient preprocessing and Fourier-Mellin transformation are performed on the corrected image to obtain the registration parameters. Eventually, the experiment results show that the method makes the image registration of Fourier-Mellin transformation not only applicable to the registration of the parallel image pairs, but also to the registration of non-parallel image pairs. What's more, the better registration effect can be obtained.
\end{abstract}

\section{INTRODUCTION}

In fact, image registration, widely used in the field of image analysis application, is the conversion of space coordinate among the images with the same scene.

The image registration method based on Fourier-Mellin transformation(Chen,1994) was first proposed by Chen and other scholars in 1994. It assumes that one picture is only rotated, scaled, and translated to get another image which is used for registration (the paper says the transformation as RST). The method obtains their relative translation by calculating the peak position, which can be get from the inverse Fourier transform of the two image cross-power spectra; the relative rotation angle and scale factors are obtained by a method similar to the calculation of the relative translation in the Log-Polar space, so the amplitude spectrum is transformed into Log-Polar space. Then, the parameters which meet RST are used to the registration between two two-dimensional images. Chen and Reddy et al. also applied the algorithm to remote sensing image, medical image, target recognition and other directions. From a lot of experiments, it's found that the method has many advantages such as high precision and strong robustness to shading change, partial occlusion, the influence of noise and so on. What's more, it has less computational complexity than other methods (Brown, 1999) and (Zitová, 2003).
However, it usually can't get the rectification cross-power spectral pulse function in many aerial images registration because of the relative distortion of the image pairs due to the relative tilt of the camera. Aiming at the above problems, a method based on the combination of central projection transformation and Fourier-Mellin transform is proposed in this paper. Combining the partial parameters of the camera relative station in the space and the collinear equation of center projection, it calculates the projection transformation matrix used to rectification the image pairs. Then the registration method based on Fourier-Mellin transform in the view of gradient preprocessing is reused to the registration between images. Not only it effectively overcomes the above problems, but also extends the applications of Fourier-Mellin transform.

\section{PROJECTION TRANSFORMATION OF NON-RELATIVE PARALLEL IMAGES}

There are many changes in perspective between the oblique view and the vertical view of the aerial images. The larger the relative angle of the cameras is, the greater the distortion of the image is. In this paper, the projection transformation matrix is deduced by the central imaging equation, by which the oblique image is corrected to image registration later.

\footnotetext{
* Corresponding author

Fund Project: National Natural Science Foundation of China (40771178)
} 

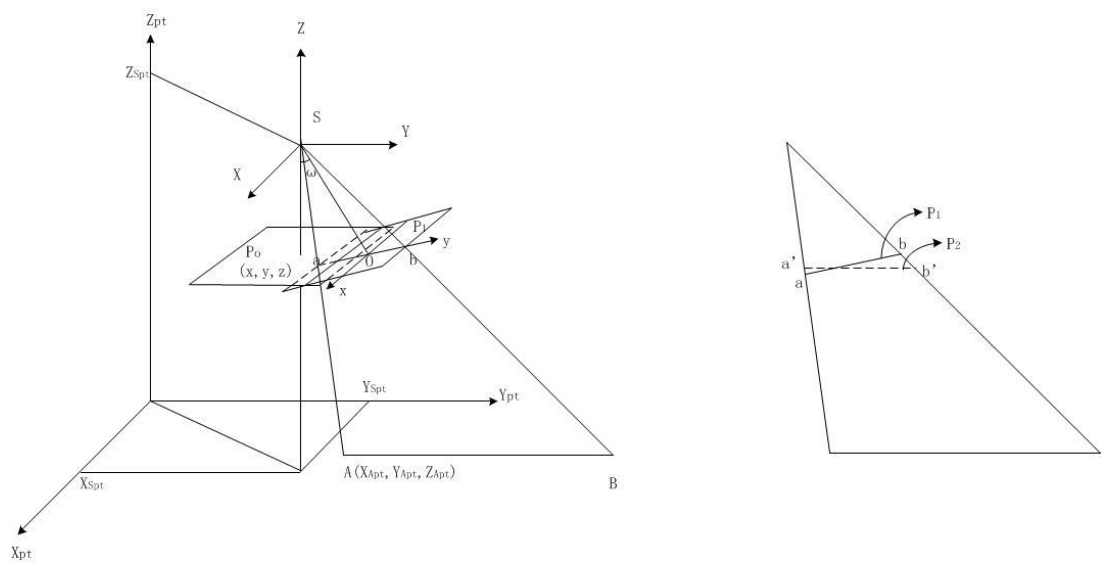

Figure 1. Non-relative parallel conformational projection relation

As shown in Figure 1, $S$ is the photography center, $A, B$ are the points on the ground, $S-X Y Z$ is image space-assisted coordinate system. The coordinate of the point $a$ is $(X, Y, Z)$ in the coordinate system. $O-x y z$ is the image space coordinate system, and the coordinate of the point $a$ is $(x, y, z)$ in the coordinate system. $D-X_{p t} Y_{p t} Z_{p t}$ is the photogrammetric coordinate system. Its $X_{p t}, Y_{p t}, Z_{p t}$ axis parallel to the axis of image space-assisted coordinate system, and have the same orientation. The coordinates of $S, A$ are $S\left(X_{S p t}, Y_{S p t}, Z_{S p t}\right)$ and $A\left(X_{A p t}, Y_{A p t}, Z_{A p t}\right)$ in the photogrammetric coordinate system. $P_{0}$ is ortho image, $P_{1}$ is tilted image, $P_{2}$ is the image that is corrected.

The relationship between the image space-assisted coordinate system and the image spatial coordinate system is shown in formula (1), where $a_{1}, a_{2}, a_{3}, b_{1}, b_{2}, b_{3}, c_{1}, c_{2}, c_{3}$ are elements of exterior orientation which are determined by the angle of camera's attitude, and $f$ is the focal length of camera. The relationship between the image space-assisted coordinate system and the photogrammetric coordinate system is shown in

$$
\left[\begin{array}{c}
\left(X_{A p t}-X_{S p t}\right) W \\
\left(Y_{A p t}-Y_{S p t}\right) W \\
W
\end{array}\right]=\left[\begin{array}{c}
\left(Z_{A p t}-Z_{S p t}\right) a_{1} \\
\left(Z_{A p t}-Z_{S p t}\right) b_{1} \\
c_{1}
\end{array}\right.
$$

As shown in Figure 1, the experiment that $P_{1}$, an oblique image, transform to $P_{2}$, a vertical image, is a transformation that a plane project to another plane. We can regard $P_{2}$ as a surface of matter, and use the formula (4) to calculate the position $a^{\prime}\left(X_{1 p t}{ }^{\prime}, Y_{1 p t}{ }^{\prime}, Z_{1 p t}{ }^{\prime}\right)$ in the photogrammetric coordinate system of the corrected image:

$$
\left[\begin{array}{c}
\left(X_{A p t}{ }^{\prime}-X_{S p t}{ }^{\prime}\right) W \\
\left(Y_{A p t}{ }^{\prime}-Y_{S p t}{ }^{\prime}\right) W \\
W
\end{array}\right]=\left[\begin{array}{ccc}
-f a_{1} & -f a_{2} & -f^{2} a_{3} \\
-f b_{1} & -f b_{2} & -f^{2} b_{3} \\
c_{1} & c_{2} & -f^{2} c_{3}
\end{array}\right]\left[\begin{array}{l}
x \\
y \\
1
\end{array}\right]=H\left[\begin{array}{l}
x \\
y \\
1
\end{array}\right]
$$

Because there is a certain angle $\omega$ only in the $y$ direction between the camera of vertical photography and the camera of oblique photography, and both the other relative angles are zero in the other two directions. $a_{1}=1, a_{2}=0, a_{3}=0, b_{1}=0$, formula (2).

$$
\begin{aligned}
& {\left[\begin{array}{c}
X \\
Y \\
Z
\end{array}\right]=\left[\begin{array}{lll}
a_{1} & a_{2} & a_{3} \\
b_{1} & b_{2} & b_{3} \\
c_{1} & c_{2} & c_{3}
\end{array}\right]\left[\begin{array}{c}
x \\
y \\
-f
\end{array}\right]} \\
& {\left[\begin{array}{c}
X_{A p t}-X_{S p t} \\
Y_{A p t}-Y_{S p t} \\
Z_{A p t}-Z_{S p t}
\end{array}\right]=\frac{Z}{Z_{A p t}-Z_{S p t}}\left[\begin{array}{c}
X \\
Y \\
Z
\end{array}\right]}
\end{aligned}
$$

Formula (3) can be deduced by the two formulas above.

$$
\left[\begin{array}{c}
X_{A p t}-X_{S p t} \\
Y_{A p t}-Y_{S p t} \\
Z_{A p t}-Z_{S p t}
\end{array}\right]=\frac{Z}{Z_{A p t}-Z_{S p t}}\left[\begin{array}{ccc}
a_{1} & a_{2} & a_{3} \\
b_{1} & b_{2} & b_{3} \\
c_{1} & c_{2} & c_{3}
\end{array}\right]\left[\begin{array}{c}
x \\
y \\
-f
\end{array}\right]
$$

In order to eliminate the $\mathrm{Z}$ in formula (3), we use $W=c_{1} x+c_{2} y-c_{3} f$. So the matrix can be translated to formula (4)

$\left.\begin{array}{cc}\left(Z_{A p t}-Z_{S p t}\right) a_{2} & -f\left(Z_{A p t}-Z_{S p t}\right) a_{3} \\ \left(Z_{A p t}-Z_{S p t}\right) b_{2} & -f\left(Z_{A p t}-Z_{S p t}\right) b_{3} \\ c_{2} & -f c_{3}\end{array}\right]\left[\begin{array}{l}x \\ y \\ 1\end{array}\right]$

$b_{2}=\cos \omega, \quad b_{3}=-\sin \omega, \quad c_{1}=0, \quad c_{2}=\sin \omega \quad$ and $c_{3}=\cos \omega$ can be calculated based on the formula of elements of exterior orientation. We replace these numbers into the formula (5), so we can get the formula (6).

$$
H=\left[\begin{array}{ccc}
-f & 0 & 0 \\
0 & -f \cos \omega & -f^{2} \sin \omega \\
0 & \sin \omega & -f \cos \omega
\end{array}\right]
$$

If the coordinate of one point is $\left(x_{0}, y_{0}\right)$ in the oblique image and it's $\left(x_{1}, y_{1}\right)$ in the corrected image, we can calculate the coordinates of each point in the corrected image by the formula (7). 


$$
\left[\begin{array}{l}
x^{\prime} \\
y^{\prime} \\
z^{\prime}
\end{array}\right]=H\left[\begin{array}{c}
x_{0} \\
y_{0} \\
1
\end{array}\right], \quad x_{1}=\frac{x^{\prime}}{z^{\prime}} ; y_{1}=\frac{y^{\prime}}{z^{\prime}}
$$

\section{GRADIENT PREPROCESSING AND FREQUENCY DOMAIN CROSS CORRELATION}

If $f_{2}(x, y)$ is the image after only shifted $\left(x_{0}, y_{0}\right)$ with respect to $f_{1}(x, y)$, then:

$$
f_{2}(x, y)=f_{1}\left(x-x_{0}, y-y_{0}\right)
$$

If the results are $F_{1}(u, v)$ and $F_{2}(u, v)$ which are corresponded to $f_{1}$ and $f_{2}$ in the frequency domain, then their relationship is as follows:

$$
F_{2}(u, v)=F_{1}(u, v) e^{-j\left(u x_{0}+v y_{0}\right)}
$$

And the reciprocal power spectrum of $f_{1}$ and $f_{2}$ is shown in formula (10)

$$
\frac{F_{1}(u, v) F_{2}^{*}(u, v)}{\left|F_{1}(u, v) F_{2}^{*}(u, v)\right|}=e^{j\left(u x_{0}+v y_{0}\right)}
$$

$F_{2}^{*}$ is the complex conjugate of $F_{2}$. It can be seen from the formula, the phase difference between the mutual power spectrum and the image is equivalent. If the inverse Fourier transform of the formula (11) is carried out, a pulse function will be formed at the space coordinate system, and the pulse peak position $\left(x_{0}, y_{0}\right)$ is the relative size of the translation between the two registered images.

\section{1 gradient preprocessing}

Gradient is the first order difference of the image, and it is also a way of usual image processing. If $f(x, y)$ is the original image, the gradient of any point $(x, y)$ in the image is a vector, then the definition as follows:

$$
\operatorname{Grad}[f(x, y)]=\left[\frac{\partial f}{\partial x} \frac{\partial f}{\partial y}\right]^{T}
$$

The gradient of the image is represented by $\operatorname{Grad}[f(x, y)]$ in the equation, referring to the rate of change in $x$ and $y$ directions. As the image is discrete, we usually use difference instead of differential, so the approximate solution can be obtained as follows:

$$
\begin{aligned}
& H G=|f(x+1, y)-f(x, y)| \\
& V G=|f(x, y+1)-f(x, y)|
\end{aligned}
$$

Where $H G$ is the horizontal gradient and $V G$ is the vertical gradient. The plural $H G+i^{*} V G$ is the information to be registered. This method is the gradient preprocessing method.

\subsection{Fourier-Mellin transform}

Assume that $t(x, y)$ is the vertical image, $i(x, y)$ is the image to be registered, and $i_{1}(x, y)$ is the corresponding part of them. It assumes that $t(x, y)$ is only rotated, scaled, and translated to get $i(x, y)$ and the parameters are $\Delta \theta,(\Delta x, \Delta y)$ and $\lambda$

$i_{1}(x, y)=t[\lambda(x \cos \Delta \theta+y \sin \Delta \theta)-\Delta x, \lambda(-x \sin \Delta \theta+y \cos \Delta \theta)-\Delta y]$

The Fourier transform is respectively performed on the left and right of the equation, and then we can get the follow formula:

$$
I_{1}(u, v)=\frac{1}{\lambda^{2}} e^{-2 \pi(u \Delta x+v \Delta y)} T\left[\frac{1}{\lambda}(u \cos \Delta \theta+v \sin \Delta \theta), \frac{1}{\lambda}(-u \sin \Delta \theta+v \cos \Delta \theta)\right]
$$

In the formula (15), $I_{1}(u, v)$ is the spectrum of the Fourier transformation of $i_{1}(x, y) ; T(u, v)$ is the spectrum of the Fourier transform of $t(x, y)$. It can be seen from equation (15), the relative displacement between the two images is present only in the phase spectrum. If we separately calculate the amplitude spectrum on both sides of formula (15), we can get:

$$
M(u, v)=\frac{1}{\lambda^{2}} M_{T}\left[\frac{1}{\lambda}(u \cos \Delta \theta+v \sin \Delta \theta), \frac{1}{\lambda}(-u \sin \Delta \theta+v \cos \Delta \theta)\right]
$$

Where $M(u, v)$ and $M_{T}(u, v)$ are respectively the amplitude spectra of $I_{1}(u, v)$ and $T(u, v)$. Convert the amplitude spectrum to the paired-polar coordinate space, and you can get:

$$
M(\lg \rho, \theta)=\frac{1}{\lambda^{2}} M_{T}(\lg \rho-\lg \lambda, \theta-\Delta \theta)
$$

$M(\lg \rho, \theta)$ is the pair-polar coordinate transformation of $M(u, v)$, and $M_{T}(\lg \rho, \theta)$ is the pair-polar coordinate transformation of $M_{T}(u, v)$. Since $\frac{1}{\lambda^{2}}$ can only affect the value, having no effect on the calculation of rotation, translation and scaling parameters, so we can ignore it. It can be seen from equation (17), the rotation and scaling parameters that exist between $t(x, y)$ and $i(x, y)$ are converted to relative translations in logarithmic-polar coordinate, i.e. $(\lg \lambda, \Delta \theta)$. The parameters can be calculated by performing a phase correlation algorithm on the amplitude spectrum of log-polar coordinates. Equation (17) is called frequency domain cross correlation transformation (Chen, 2009) and (Jiao J C, Zhao B J, Zhou G., 2010).

\section{The Algorithmic process}

According to the analysis in the previous section, the steps of this algorithm are as follows:

Let $t(x, y)^{\prime}$ be the vertical image and $i(x, y)^{\prime}$ be the image to be registered 
(1) $t(x, y), i(x, y)$ can be get through projection transformation.

(2)The horizontal gradients and vertical gradients $H G_{1}, V G_{1}$ and $H G_{2}, V G_{2}$ of the two images are calculated separately, and then we can get the preprocessed image $G_{1}, G_{2}$.

(3) Fourier transformation can be performed on $G_{1}, G_{2}$ respectively, so we can get $T(u, v)$ and $I(u, v)$.

(4) Calculate the amplitude spectra $M(u, v)$ and $M_{T}(u, v)$ of $T(u, v)$ and $I(u, v)$ respectively.

(5)The amplitude spectrum is subjected to high-pass filtering, and then the spectrum is converted to the log-polar coordinates to obtain $M(\lg \rho, \theta)$ and $M_{T}(\lg \rho, \theta)$;

(6) The relative displacement parameter between $M(\lg \rho, \theta)$ and $M_{T}(\lg \rho, \theta)$ is obtained by using the phase correlation algorithm, and the rotation angle $\Delta \theta$ and the scaling coefficient $\lambda$ are obtained.

(7) According to $\Delta \theta$ and $\lambda$, the image to be registered is transformed to the image $I^{\prime}(x, y)$ which only has the relative translation parameter.

(8) For $t(x, y)$ and $I^{\prime}(x, y)$, using the phase correlation algorithm, the translation parameter $(\Delta x, \Delta y)$ is obtained

\section{EXPERIMENT AND RESULT ANALYSIS}

In order to verify, this paper will experiment on the images which include typical objects in the remote sensing data of the Resource No. 3 such as buildings, roads, lakes, in Figure 2, Figure 3, Figure 4. And the experiment is compiled by using matlab. The results of the comparison from the cross-power spectrum results and the final results of the registration are presented the usefulness of the proposed method.

In this paper, the RMSE method is used to evaluate the experiment. RMSE is defined as:

$$
R M S E=\sqrt{\frac{1}{N}\left(\sum_{i=1}^{N}\left\|(x, y)_{i}-\left(x^{\prime}, y^{\prime}\right)\right\|^{2}\right)}
$$

The number of the characteristic object of image is $\mathrm{N},(\mathrm{x}, \mathrm{y})$ is the position of the center of the characteristic object in the standard image, and $\left(\mathrm{x}^{\prime}, \mathrm{y}^{\prime}\right)$ is the position of the center of the characteristic object in the image after registration.

Table 1.Comparison of RMSE values after registration of different objects

\begin{tabular}{ccc}
\hline Feature type & $\begin{array}{c}\text { Traditional FMT } \\
\text { registration }\end{array}$ & $\begin{array}{c}\text { The method of } \\
\text { this article }\end{array}$ \\
\hline Building & 3.466 & 0.608 \\
Road & 2.768 & 0.847 \\
Lake & 1.358 & 0.522 \\
\hline
\end{tabular}

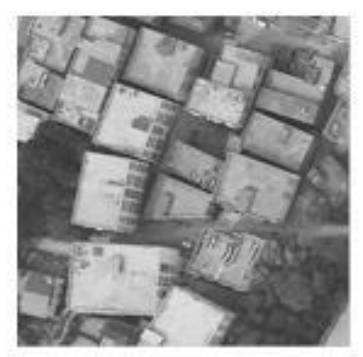

(a) Left

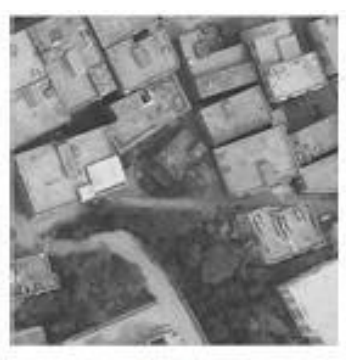

(b) Right

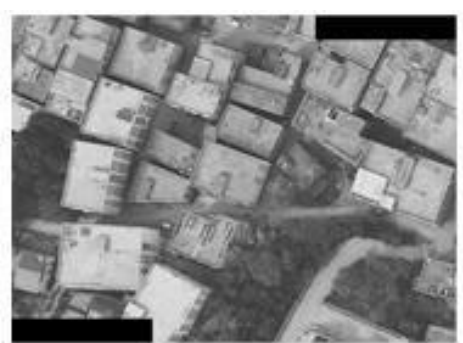

(c) Registered image

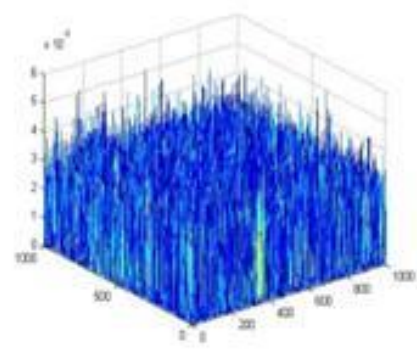

(d) The image has not been pre-processed by the cross-correlation image.

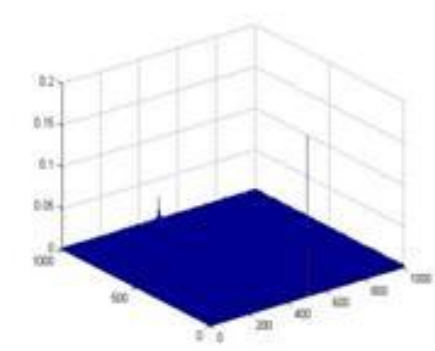

(e) The image is pre-processed by the cross-correlation image.

Figure 2. Result1 


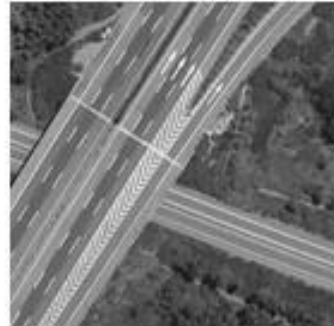

(a) Left

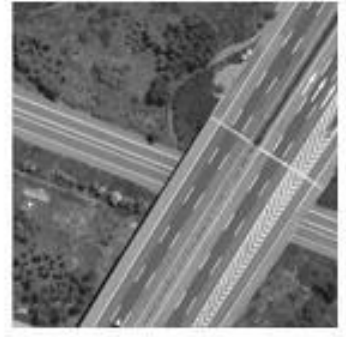

(b) Right

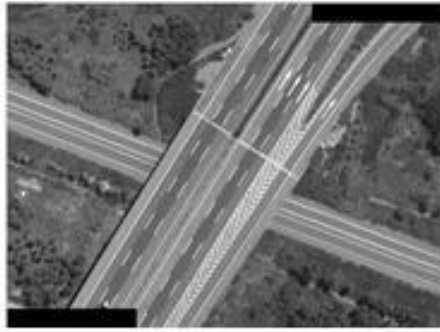

(c) Registered image

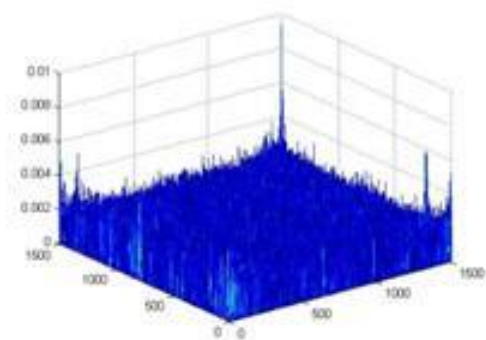

(d) The image has not been pre-processed by the cross-correlation image.

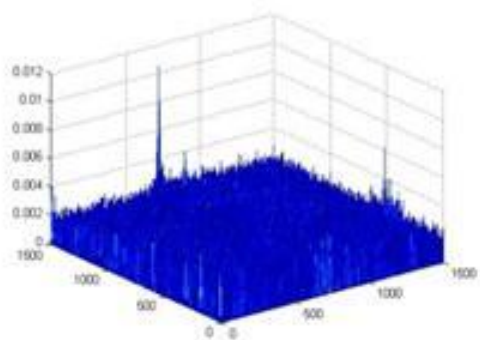

(?) cross-correlation image.,

Figure 3. Result2

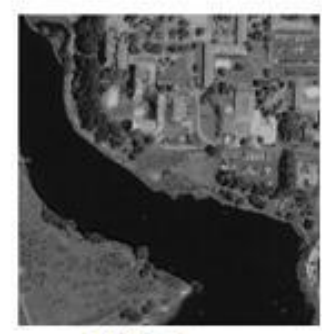

(a) Left

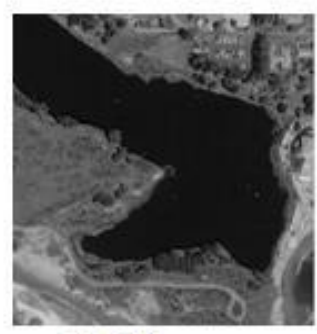

(b) Right

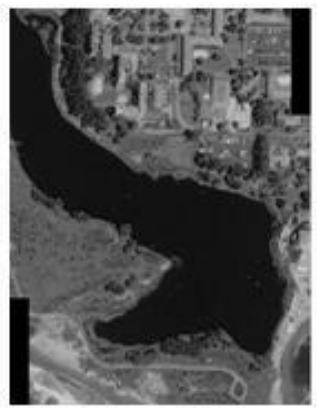

(c) Registered image

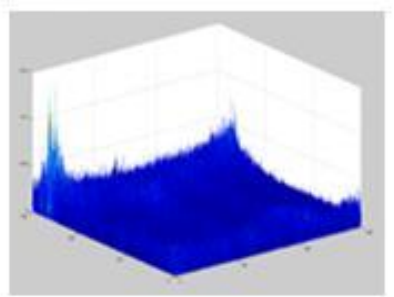

(i) The image has not been pre-processed by the cross-correlation image.

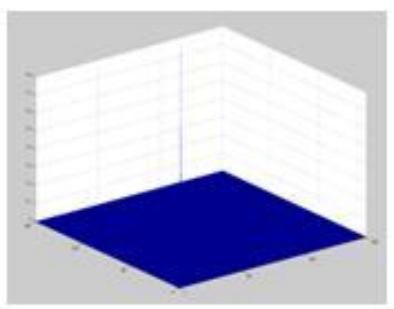

(e) The image is pre-processed by the cross-correlation image.

Figure 4. Result3

From Fig. 2, Fig. 3 and Fig. 4, their graphs (a) and (b) are the original graphs. We can see that the cross-power spectra, Figure $\mathrm{d}$,obtaining by directly performing Fourier-Mellin transform image registration without projection transformation and gradient preprocessing. And the cross-power spectrum of roads and lakes is inaccurate, what's more the cross-power spectrum peaks of buildings are not unique. So if the image is registered in accordance with the traditional Fourier-Mellin transformation for such images will not be accurately registered.
However, after the improvement of this method, the obtained cross-power spectrum (Figure e) can be a good peak. What's more the comparison of Table 1 can be used to objectively illustrate the accuracy of the method with the oblique image registration is higher than the traditional Fourier Merlin algorithm. So in this paper, we can get relatively accurate registration parameters which can be used to register the images well for the registration process of non-parallel image pairs with relative tilting cameras. 


\section{CONCLUSION}

In this paper, the horizontal images registration method based on the Fourier-Mellin transform in use of global spectrum is extended to relatively oblique images registration method. A new method based on projection transformation and gradient preprocessing Fourier-Mellin transform of the image registration method is proposed. Because this method makes Fourier-Mellin transform image registration not rigidly limited to the limitations of the image itself at some extent, it greatly extends the application of Fourier-Mellin transform image registration. What's more, a new solution is proposed for aviation images registration.

\section{REFERENCES}

[1]Chen Q, Defrise M, Deconinck F. 1994, Symmetric Phase-Only Matched Filtering of Fourier-Mellin Transforms for Image Registration and Recognition[J]. Pattern Analysis \& Machine Intelligence IEEE Transactions on, 16(12):1156-1168. [2] Reddy B S, Chatterji B N. 1996, An FFT-based technique for translation, rotation, and scale-invariant image registration[J]. IEEE Transactions on Image Processing, 5(8):1266-1271.

[3] Brown L G. 1999, A Survey of Image Registration Techniques. ACM Computing Surveys 24: 325-376[J]. Acm Computing Surveys, 24(4):325-376.

[4] Zitová B, Flusser J. 2003, Image registration methods: a survey[J]. Image \& Vision Computing, 21(11):977-1000.

[5] Xiao Ming L I, University S, Taiyuan. 2006, An Image Registration Technique Based on Fourier-Mellin Transform and Its Extended Applications[J]. Chinese Journal of Computers, 29(3):466-472.

[6] Chen F. 2009. Image Matching System Based on Fourier-Mellin Method[J]. Journal of Capital Normal University,].

[7] Jiao J C, Zhao B J, Zhou G. 2010, A fast image registration algorithm based on Fourier-Mellin transform for space image[J]. Acta Armamentarii, 31(12):1551-1556. 